\title{
A novel method for EMG decomposition based on matched filters
}

\author{
Ailton Luiz Dias Siqueira Júnior*, Alcimar Barbosa Soares
}

\begin{abstract}
Introduction: Decomposition of electromyography (EMG) signals into the constituent motor unit action potentials (MUAPs) can allow for deeper insights into the underlying processes associated with the neuromuscular system. The vast majority of the methods for EMG decomposition found in the literature depend on complex algorithms and specific instrumentation. As an attempt to contribute to solving these issues, we propose a method based on a bank of matched filters for the decomposition of EMG signals. Methods: Four main units comprise our method: a bank of matched filters, a peak detector, a motor unit classifier and an overlapping resolution module. The system's performance was evaluated with simulated and real EMG data. Classification accuracy was measured by comparing the responses of the system with known data from the simulator and with the annotations of a human expert. Results: The results show that decomposition of non-overlapping MUAPs can be achieved with up to $99 \%$ accuracy for signals with up to 10 active motor units and a signal-to-noise ratio (SNR) of $10 \mathrm{~dB}$. For overlapping MUAPs with up to 10 motor units per signal and a SNR of $20 \mathrm{~dB}$, the technique allows for correct classification of approximately $71 \%$ of the MUAPs. The method is capable of processing, decomposing and classifying a $50 \mathrm{~ms}$ window of data in less than $5 \mathrm{~ms}$ using a standard desktop computer. Conclusion: This article contributes to the ongoing research on EMG decomposition by describing a novel technique capable of delivering high rates of success by means of a fast algorithm, suggesting its possible use in future real-time embedded applications, such as myoelectric prostheses control and biofeedback systems.
\end{abstract}

Keywords: EMG decomposition, MUAPs classification, Matched filters.

\section{Introduction}

An electromyographic (EMG) signal is a measure of the electrical manifestation of a muscle contraction and the result of the asynchronous summation of basic signals known as motor unit action potentials (MUAPs) (Parsaei and Stashuk, 2011; 2013).

The waveforms and rates of occurrence of MUAPs allow for the identification of motor disturbances (Han et al., 2005; McGill and Marateb, 2011; Pino et al., 2008) and provide information on the underlying mechanisms of the neuromuscular control system (Barboi and Barkhaus, 2004). However, extensive processing is required to distinguish individual MUAPs from EMG signals. This process, known as "decomposition", generally involves the following steps (Parsaei et al., 2010; Stashuk, 2001): (a) EMG data acquisition - usually by means of multiple electrodes; (b) pre-processing - to improve the signal-to-noise ratio and highlight MUAPs; (c) windowing - selection of the regions of EMG activity; (d) feature extraction - the EMG data are processed and transformed into numerical features, generally a multidimensional array; and (e) pattern recognition - detected MUAPs with similar features are separated into groups, representing the action potentials of specific motor units (MUs). The last step assumes that MUAPs from the same MU would share a distinct signature that accounts for similarities in the extracted features.

To date, a number of methods for EMG decomposition have been described in the literature (Andrade et al., 2007; Christodoulou and Pattichis, 1995; De Luca et al., 2006; Sá et al., 2014; Fang et al., 1999; Florestal et al., 2006; Glaser et al., 2013; Lefever and De Luca, 1982; Mambrito and De Luca, 1984; Marateb et al., 2011; McGill and Marateb, 2011; McGill et al., 1985; Nawab et al., 2008; Rasheed et al., 2008; Ren et al., 2006; Stashuk; 2001; Xu et al., 2001; Winslow et al., 2009). Nevertheless, the vast majority of those methods are limited to laboratory research and are usually based on specific instrumentation and complex algorithms that require large amounts of data to train the mathematical models (Glaser et al., 2013; Holobar et al., 2009). Additionally, traditional methods for EMG decomposition may take many hours or even days to process large sets of data, such as those obtained in the experiments reported by Kleine et al. (2012), Winslow et al. (2009), Zhou et al. (2011) and Zijdewind and Thomas (2012), that use up to 24 hours of multichannel EMG data to study different aspects of the neuromuscular process in paraplegic patients. 
The increasing interest in the application of EMG signal decomposition in areas such as prosthesis control, human-machine interface and medical diagnosis is driving researchers to develop better and more accurate decomposition strategies, preferably ones capable of online processing without demanding complex instrumentation.

Matched filters is a technique used in areas such as radar and communication systems with great success (Lathi and Ding, 2009; Turin, 1960), relying on symbols (pulses) transmitted via noisy channels that are detected and classified in search of a message in real time. Because this bears strong analogy to the detection of MUAPs in EMG signals, the authors hypothesize that this approach can also be used as a strategy to perform fast and reliable decomposition. Furthermore, another feature of matched filters may also play an important role for real-world EMG decomposition: the capacity to maximize the output signal-to-noise ratio. This could prove particularly useful for real-world applications where high signal-to-noise ratios are not always achievable. Therefore, this technique may allow for the design of a method that rapidly decomposes EMG signals that does not rely upon complex instrumentation, such as multi-channel electrode arrays, paving the way for the use of MUAP-based control algorithms in devices such as myoelectric prostheses, where they still cannot be applied due to the extensive computing required by current EMG decomposition algorithms.

It is important to highlight that the expression "matched filter" has also been used in the literature to describe other specific methods for EMG processing. For instance, Studer et al. (1984) use the expression "matched filter" to describe a method used to adapt signal templates of a filter bank to detect MUAPs in EMG data. However, their technique is based on Kalman filters, which is considerably different from what we proposed in this article. To the best of our knowledge, this is the first time matched filters, as described in this paper, are used for surface EMG decomposition.

\section{Methods}

\section{EMGD-MF: a novel method for EMG decomposition based on matched filters}

Our method for EMG decomposition can be expressed by four main units (Figure 1): (a) a bank of matched filters, (b) a peak detector, (c) a classifier and (d) an overlapping resolution module. The input to the system is a single EMG channel that is first processed by a parallel bank of matched filters (Figure 1a), where each filter is responsible for the detection of an individual MUAP. The outputs of the filters are then biased by a specific value, depending on the features (templates) of each filter, and their maxima (peaks) are calculated (Figure 1b). At this stage a validation algorithm is also used to eliminate local maxima associated with noise and responses to inputs too far from the filter's template. Finally, a classifier (Figure 1c) selects the output that best represents the spike at the input of the bank of filters. To minimize errors caused by overlapping MUAPs, the set of MUAPs detected by the classifier is sent to a synthesizer to construct an EMG signal represented by the previously detected MUAPs, which is then subtracted from the raw EMG signal (Figure 1d).

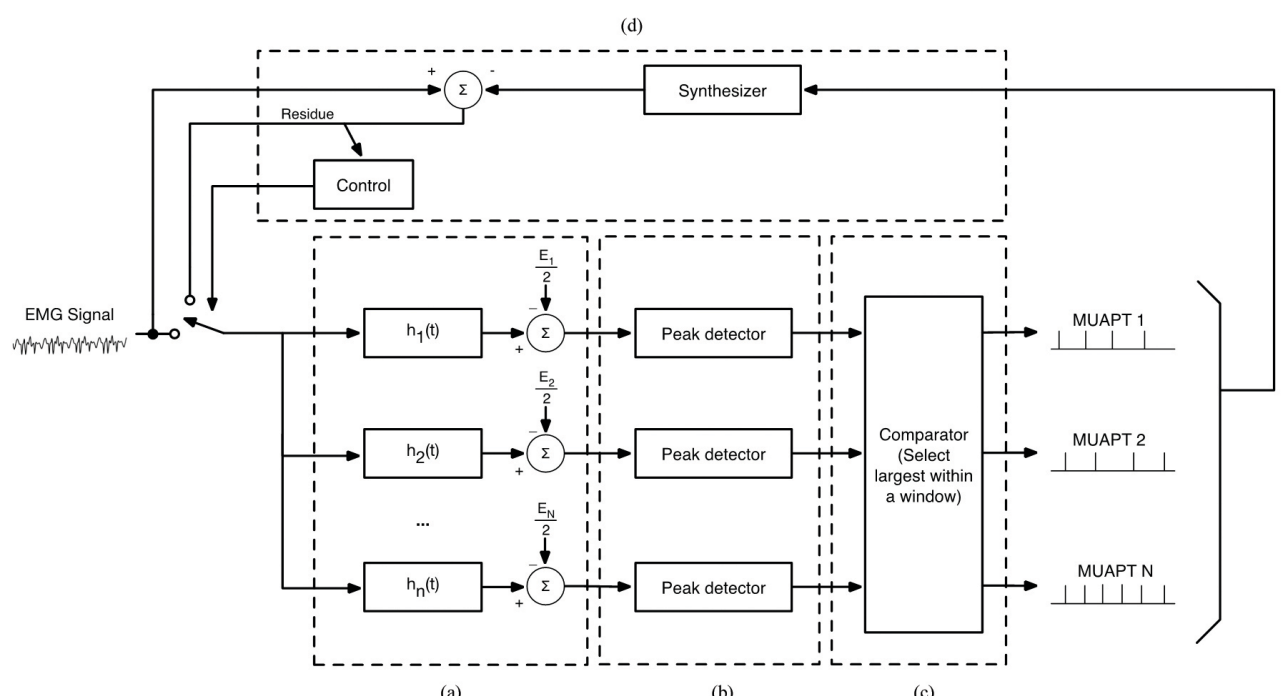

Figure 1. A block diagram of the proposed EMGD-MF system: (a) bank of matched filters; (b) peak detector; (c) classifier; (d) overlap resolution. 
A control block evaluates the energy of the residue and determines if it is to be sent to the input of the system, allowing for the detection of MUAPs that were not identified in the first iteration.

To illustrate the operation of the units, throughout this section, a synthetic EMG signal with three pseudo-MU spikes without overlapping, as shown in Figure 2a, will be used.

\section{Bank of matched filters}

A matched filter is the optimal linear filter for maximizing the output signal-to-noise ratio (SNR) of a signal in the presence of stochastic noise. A filter is said to be matched to (template) if its impulse response is given by (Lathi and Ding, 2009; Turin, 1960):

$h(t)=k \cdot g(\tau-t)$

where and are arbitrary constants (because $k$ behaves like a gain applied to the template, it can be set to 1 for simplicity; similarly, can be set to the duration of ).

Therefore, if we use this filter to detect the presence of a known signal $g(t)$ mixed with Gaussian noise $n(t)$ in the input, its output would show values between two extremes: (a) minimum - if $g(t)$ is not present $(s(t)=n(t))$ - and (b) maximum - if $s(t)=g(t)+n(t)$.
Equation 2 shows a model for EMG signals proposed by De Luca et al. (2006):

$$
E M G(t)=\sum_{j=1}^{N_{m}} M U A P T_{j}(t)+n(t)
$$

where is the j-th MUAP train, is the number of active MUs and represents noise.

The trains of MUAPs can be modeled as:

$$
\operatorname{MUAPT}_{j}(t)=\sum_{k=1}^{M j} M U A P_{j k}\left(t-\delta_{j k}\right)
$$

where corresponds to the train of MUAPs of the $j$-th MU, corresponds to the waveform of the k-th spike of the $\mathrm{j}$-th MU, corresponds to the number of spikes generated by $\mathrm{MU}_{\mathrm{j}}$ and represents the time of each spike. Because the waveforms of the various firings of $\mathrm{MU}_{\mathrm{j}}$ are similar to each other, we can design a template that represents the mean waveform (template for a filter) and use it to identify the presence and the time when the $\mathrm{MU}_{\mathrm{j}}$ fired, which are the key elements we are looking for.

As described earlier, an EMG signal is composed of various MUAP trains (MUAPTs). Therefore, a real-time decomposition system should be designed as a bank of filters working in parallel, in which each isolated filter is designed for each MU present

a) EMG Signal
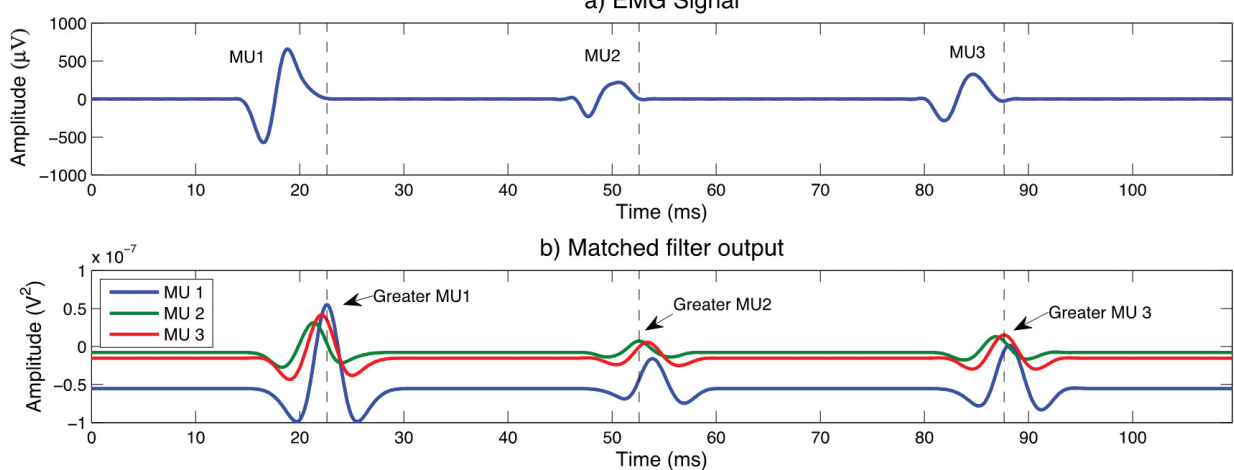

c) Possibile spikes

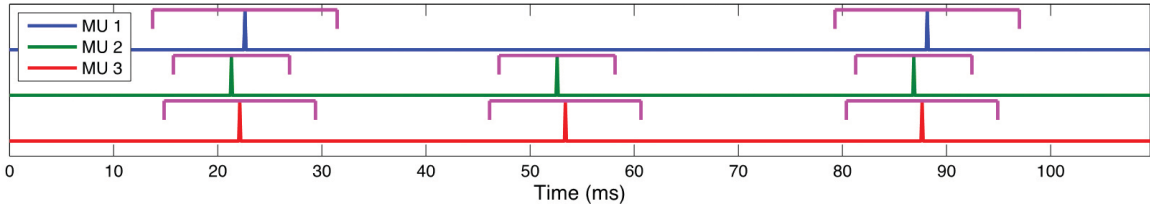

d) Classifier output

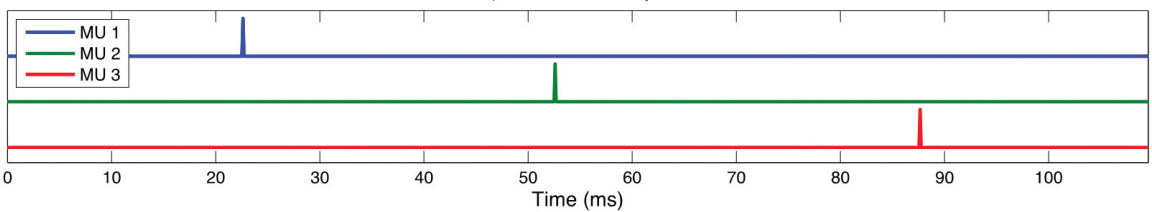

Figure 2. The EMGD-MF processing a simulated signal with three MUs firing sequentially: (a) input signal; (b) output from the bank of filters; (c) output from the peak detector with the possible spikes and the analysis window used for classification; (d) output from the classifier with the estimated spikes. 
in the signal. This strategy was previously used in telecommunication to classify pulses in $\mathrm{M}$-ary receivers. The overall process for $\mathrm{M}$-ary receivers is described in Lathi and Ding (2009) and can be summarized as follows: (1) in the receiver, the input signal is applied to a bank of matched filters, where each filter is designed to detect a specific symbol, (2) the outputs are biased by a distinct value $\left(a_{i}\right)$, (3) the outputs are sampled when they reach their maximum value (peak) and (4) the outputs are compared to estimate the symbol received, i.e., the template related to the filter with the highest output value. Steps 1, 3 and 4 are quite straightforward; however, we must define the bias to be applied to the output of each matched filter. This is an important element because every filter will respond to every pulse, regardless if it is its base pulse $(g(t))$ or not. Equation 4 defines the bias for a pulse $i$, where is a constant representing white noise, is the probability of the pulse (symbol) associated with a filter occurring in the signal, and is the energy of the template used in the design of the filter (Lathi and Ding, 2009).

$a_{i}=\frac{1}{2}\left[N_{0} \ln P\left(m_{i}\right)-E_{i}\right]$

In the case where the probability of receiving a certain symbol is the same as that for all other pulses, is the same for all symbols and, therefore, can be removed from the equation when we are looking for differences among the outputs (as in the M-ary systems):

$a_{i}=-\frac{E_{i}}{2}$

The EMG signal is applied to the bank of matched filters (Figure 1a), and a bias value is added to the outputs of each filter, as defined in Equation 5. To construct a filter, one must first model the pulse $(g(t))$ used to estimate the impulse response of the filter. As explained earlier, we can model $g(t)_{j}$ as a mean template waveform of the MUAPs associated with $\mathrm{MU}_{\mathrm{i}}$. For simulated signals, for instance, $g(t)_{j}$ could be modeled as the average of all simulated spikes of a $\mathrm{MU}_{\mathrm{i}}$, as described by Andrade et al. (2005). However, for real EMG signals, one must observe a certain amount of data before templates can be modeled and used to fit the filters. Such a task may be conducted by an expert in MUAP segmentation, who would manually mark and group the occurrences of all MUAPs, or by means of an automated tool, such as the ones described by Andrade et al. (2005), Parsaei and Stashuk (2011; 2013).

In this work, $g(t){ }_{j}$ was obtained as follows: a) for simulated data, the waveform of the first MUAP fired by the simulator for $\mathrm{MU}_{\mathrm{j}}$ was used as $g(t)_{j}$; (b) for the real surface EMG signal, the first 5 seconds of data were analyzed by a human expert, and $g(t)_{j}$ was modeled as the average waveform of the MUAPs fired by $\mathrm{MU}_{\mathrm{j}}$.

Once the templates $\left(g(t)_{j}\right)$ are defined, the online automated process can begin. Nevertheless, it is very likely that the initial set of filters does not contain a representation for every possible MU in the signal and false-positives may occur. This is a trade-off for any EMG decomposition system described in the literature to date.

Figure $2 b$ shows the response of a bank of matched filters designed to detect the MUAPs shown in Figure 2a. Note that the outputs of the filters are biased and, for every spike, all filters respond with a peak slightly shifted in time due to differences in the MUAPs' waveforms.

\section{Peak detector}

This stage (Figure 1b) is responsible for the detection of maxima in the output of each filter. To minimize errors caused by high frequency oscillations, the outputs of the filters are first smoothed by a 3rd order moving average filter. Local maxima are computed as described in Equation 6.

$m[i]=\left\{\left(f o[i-1]-f_{o}[i]<0\right) A N D\left(f o[i+1]-f_{o}[i]<0\right)\right\}$

where is a vector with the smoothed samples from the output of the matched filter and is the output vector with as many elements as that have values equal to 0 or 1 , where 1 indicates a local maximum at that position in .

The amplitudes of the detected peaks are then evaluated, and those showing negative values or values lower than the bias level of the associated filter are rejected (Figure 2c). This process seeks to avoid local maxima associated with low amplitude peaks resulting from noise and low outputs associated with responses to inputs too far from the filter's template symbol.

\section{Classifier}

As shown in Figure 2c, whenever a MUAP is presented at the input of the filters, there is a high probability that more than one filter will output a non-zero response. To estimate the most probable MU responsible for the correct output, the classifier applies a validation algorithm to every possible spike found by the peak detectors. Candidate peaks are sequentially tested, beginning with the first candidate peak from the first filter. A region of analysis (shown as horizontal bars above the peaks in Figure 2c) is defined around each detected peak $(D i)$ with a width equal to twice the duration of the MUAP that originated the filter. 
The peaks detected in the outputs of all filters within this region of analysis are compared with each other; if the output from the filter that originated $D i$ has the highest amplitude, then $D i$ is maintained for further testing; otherwise, it is discarded (indicating that another peak exists with a higher probability of being associated with the correct output). This process is repeated for all spikes, and the final result is illustrated in Figure 2d.

\section{Improving the resolution for overlapping peaks}

The response of the classifier for overlapping MUAPs may vary from not detecting any MUAP to partial detection (Stashuk, 2001). In the case of partial detection, assuming that a residue obtained by the subtraction of the detected MUAP from the EMG signal contains information about the remaining overlapping MUAPs, we can increase the probability of detecting them by feeding the residue back into the system and repeating the process until its energy reaches a pre-defined minimal value. This technique is known as the "peel-off superposition resolution method" (Winslow et al., 2009). Next, we demonstrate how the technique is incorporated into our method.

Equation 7 describes a model for an EMG signal window composed of overlapping MUAPs (De Luca et al., 2006; Parsaei et al., 2010; Stashuk, 2001), where denotes the waveform of the EMG signal, is a firing of $\mathrm{MU}_{\mathrm{i}}$, is the time fires, represents the number of MUs that comprise the overlap, and denotes the noise.

$$
E M G_{O v}=\sum_{j=1}^{N_{m}} M U A P_{j}\left(t-\tau_{j}\right)+n(t)
$$

Assuming that is the input to the bank of matched filters and the responses of filter for a certain window of analysis $(50 \mathrm{~ms})$ are classified as correct spikes, we can reconstruct an EMG signal () based on those spikes (Figure 1d):

$$
E M G_{\operatorname{Rec}}=\sum_{i=1}^{N} h_{i}\left(T_{i}-t-\tau_{i}\right)
$$

where $N$ is the number of spikes detected by the simulator within the window of analysis, is the impulse response for the $\mathrm{i}$-th detected spike, is the duration of the $\mathrm{i}$-th spike and is the time it was detected.

By subtracting (8) from (7), we obtain the residue $R(t)$ :

$R(t)=E M G_{O v}-E M G_{\operatorname{Rec}}$

Assuming that waveforms of the filter's templates carry strong similarities to the $M U A P_{s}$ in , the overlappings due to the MUAPs in would now be removed from the residue. Hence, by feeding $R(t)$ back into the system, we increase the probability that new MUAPs are detected.

A control block (Figure 1d) decides whether the original EMG signal or the residue will be sent to the input of the system. In the first iteration, the EMG signal is the input, and then, the residue is fed back into the filters until a stopping condition is achieved. Because we expect the residue's energy to reduce as new MUAPs are detected, the process ends when its energy does not show any significant change between consecutive iterations or increases due to, for instance, a false positive.

\section{Testing experiments}

\section{Experiments using simulated data}

Simulated signals were chosen as the gold standard to evaluate the system's performance. By controlling the number of active MUs and their firings, it is possible to achieve precise comparisons between the responses of the decomposition system and the actual data.

An EMG signal simulator, proposed by Andrade et al. (2007) was used to generate the simulated EMG data. Instead of using synthetic MUAP waveforms, this simulator uses approximately 1000 real MUAP waveforms digitized at a rate of $10,040 \mathrm{~Hz}$ and clustered into 15 MUs. The simulator allows for various configurations, such as different numbers of active MUs, firing rates and signal-to-noise ratios. In addition to the actual simulated EMG signal, the simulator also returns the timing and identification of all MUAPs.

A set of 480 simulated EMG signals (30 seconds each) was generated and divided into twelve groups of 40 signals each: (GS1) - \#MU $=3$ to 5 , $\mathrm{SNR}=10 \mathrm{~dB}$; (GS2) - \#MU $=3$ to $5, \mathrm{SNR}=20 \mathrm{~dB}$; (GS3) - \#MU $=6$ to $8, \mathrm{SNR}=10 \mathrm{~dB}$; (GS4) - \#MU $=6$ to $8, \mathrm{SNR}=20 \mathrm{~dB} ;(\mathrm{GS} 5)-\# \mathrm{MU}=9$ to $10, \mathrm{SNR}$ $=10 \mathrm{~dB}$; and (GS6) $-\# \mathrm{MU}=9$ to $10, \mathrm{SNR}=20 \mathrm{~dB}$. Signals in groups GS7 to GS12 were created similar to GS1 to GS6, but we allow for random MUAP overlapping, whereas for GS1 to GS6, no overlapping was allowed. Note that a 'minimum' number and a 'maximum' number of active MUs were defined for each group. This tells the simulator to randomly select a set of MUs for the group and randomly use anything from the 'minimum' to 'maximum' of those MUs to generate each EMG signal. Additionally, in groups GS7 to GS12, on average $42 \%$ of all observed events (MU spikes) referred to overlappings of 2 to 8 MUs; the remaining $52 \%$ of the events referred to MUs firing in isolation. 


\section{Experiments with recorded data}

EMG data from the public EMGLAB (http://emglab.net/) databank were used (Hogrel, 2014). One surface Laplacian electrode (5 mm inter-electrode distance) positioned over the brachial biceps was used to collect the chosen signal under low levels of isometric contraction. The signal was band-pass filtered $\left(10 \mathrm{~Hz}-1 \mathrm{kHz}, 10^{\text {th }}\right.$ order Butterworth) and sampled at $10 \mathrm{kHz}$ for 120 seconds. The curator of that database manually marked and classified the spikes of the MUs. Four active MUs were identified, and the annotations (MUs and spikes) were marked on the waveform.

The template for each filter $\left.(g(t))_{j}\right)$ was modeled as the average of the MUAPs' waveforms marked by the expert for each MU during the first 5 seconds of data, e.g., the first 5 seconds of data were used to generate the template for each filter and the remainder was used for testing.

\section{Performance indexes}

The following indexes were used to evaluate the performance of the EMGD-MF system:

- Correct classification rate:

$$
C C=\frac{T P}{N_{\text {Sig }}} \times 100
$$

- Precision:

$$
P=\frac{T P}{N_{\text {meth }}} x 100
$$

- False positive rate:

$$
F P_{R}=\frac{F P}{N_{\text {Sig }}} \times 100
$$

- False negative rate:

$$
F N_{R}=\frac{F N}{N_{\text {Sig }}} \times 100
$$

where $T P$ is the number of spikes correctly detected by the EMGD-MF method (true positive $=$ a correct detection of a spike from a specific MU), is the number of spikes actually present in the signal, is the number of spikes detected by the method, $F P$ is the number of false positives and $F N$ is the number of false negatives.

A spike is considered valid if it coincides with the actual firing of a MU and if it occurred at the same time or within $\pm 2.5 \mathrm{~ms}$. This tolerance refers to one half of the typical duration of the fastest MUAPs found in skeletal muscles (Florestal et al., 2006).

\section{Processing window}

For both simulated and real EMG data, the signals were processed by the EMGD-MF system using $50 \mathrm{~ms}$ windows (rectangular) with $25 \mathrm{~ms}$ overlap. The length of the processing window was set to be twice the duration of the slowest MUAPs found for skeletal muscles (Florestal et al., 2006). Window overlapping is important to avoid errors at the boundaries of the processing window, where MUAP waveforms might be cropped.

\section{Results}

\section{Simulated data}

All 480 simulated signals were decomposed by the system. To illustrate the process, Figure 3 shows a portion of a simulated signal with 5 active overlapping MUs and a SNR of $10 \mathrm{~dB}$. A cross is drawn when the simulator generates a spike (MUAP), and a circle is drawn when it is detected by the method. Colors are used to represent different MUs. A cross drawn inside a circle of the same color indicates the precise detection of a MUAP. As shown, the method was capable of detecting the majority of the spikes within the analysis window, failing only to detect the first "wave" composed of overlapping MUAPs from MUs 1 and 5 , resulting in a false detection of a spike from MU 2 (most likely because those two MUAPs have very similar shapes and both MUs fired almost exactly at the same time, resulting in a waveform very similar to MUAPs from MU 2).

Table 1 shows the mean and standard deviation of each evaluation index calculated for the simulated signals with and without overlapping MUAPs. When overlapping did not occur, the method correctly classified $97.32 \%$ to $99.55 \%$ of the MUAPs found in signals with up to 10 active MUs in the presence of high levels of noise. When decomposing simulated EMG signals with overlapping MUAPs, the method correctly classified more than $90 \%$ of those spikes for up to $5 \mathrm{MUs}$ in situations with considerable noise; however, as expected, the performance deteriorates with increases in the number of active MUs and noise intensity.

Figure 4 shows the results of the decomposition of 30 seconds of a simulated signal with 4 active MUs and random overlapping. The shimmer plots of the MUAPs' waveforms and the average waveform for each MU are shown on the left. The histograms for the spikes of those MUs are shown in the center, and the MUAP trains for the first 20 seconds of data are shown on the right. As expected, the histograms for the inter-discharge intervals (IDIs) of the 4 MUs have an approximately Gaussian shape (De Luca and Hostage, 2010). The mean firing rate for each MU, calculated as the maximum of its IDI density function (Kernel Density Estimation), shows good agreement with the actual rate calculated from the simulated signals, displayed as a vertical dotted red line over the histogram (mean errors between $0.71 \%$ and $3.28 \%$ for this signal). 


\section{Real surface EMG data}

Figure 5 illustrates the response of the EMGD-MF system when decomposing a small sample of a real surface EMG signal. The events (MUAPs) annotated by the specialist are shown as crosses, and those detected by the EMGD-MF are shown as circles. Visual inspection shows acceptable agreement between the systems' output and the specialist's annotations for MUs 1 (blue), 2 (red) and 3 (green). Divergences can be found with regard to MU 4 (black). A closer inspection

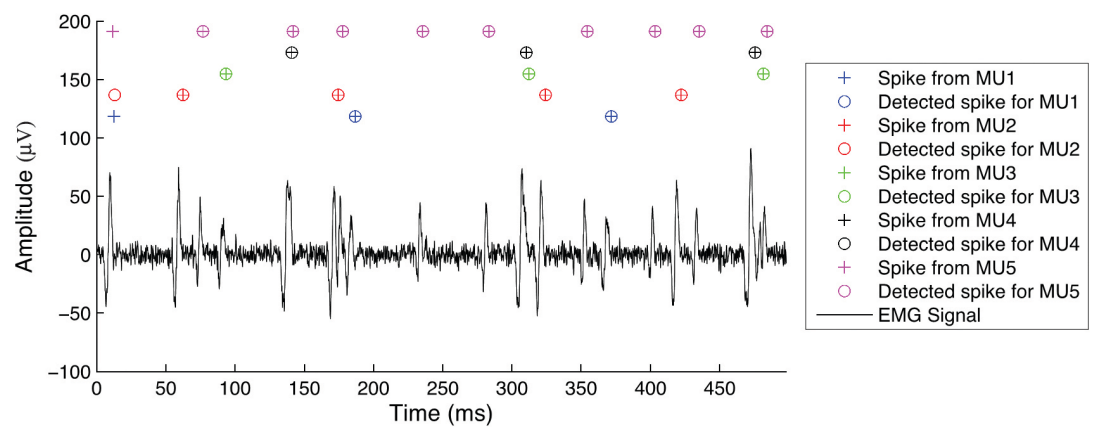

Figure 3. The EMGD-MF processing a simulated EMG signal with 5 active overlapping MUs and a SNR of $10 \mathrm{~dB}$. The actual spikes are marked with a cross, and those detected are represented as circles.
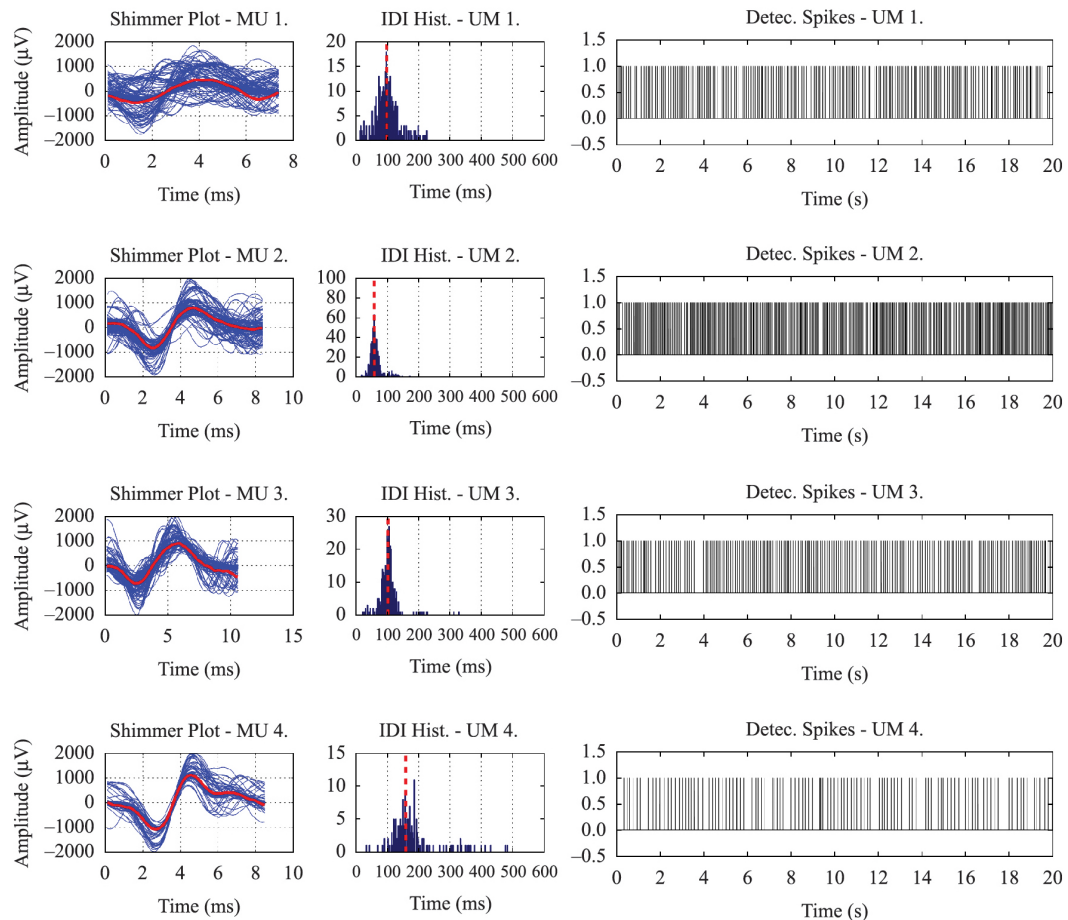

Figure 4. Decomposition of a simulated signal with 4 active overlapping MUs and a SNR of $20 \mathrm{~dB}$. The shimmer plot, the histogram of the inter-discharge/spikes intervals (IDIs) and a sample of the pulse train for each MU are presented in the left, center and right columns, respectively. The vertical dotted red lines on the histograms show the actual average of the IDIs obtained from the simulator.

Table 1. Mean and standard deviation of the evaluation indexes for the response of the EMGD-MF system when decomposing 480 simulated signals with and without MUAP overlap and different noise levels.

\begin{tabular}{llcclccccc}
\hline \multirow{2}{*}{ MUs } & \multirow{2}{*}{ SNR } & \multicolumn{3}{c}{ Without MUAP Overlap (\%) } & \multicolumn{3}{c}{ With MUAP Overlap (\%) } \\
\cline { 3 - 9 } & & CC & P & \multicolumn{1}{c}{ FPR } & \multicolumn{1}{c}{ FNR } & CC & \multicolumn{1}{c}{ P } & FPR & FNR \\
\hline $3-5$ & 20 & $99.55(0.58)$ & $99.10(3.80)$ & $1.08(4.67)$ & $0.45(0.58)$ & $92.78(5.21)$ & $95.10(8.22)$ & $5.64(11.73)$ & $7.22(5.21)$ \\
$3-5$ & 10 & $99.15(1.57)$ & $97.00(11.66)$ & $6.39(29.66)$ & $0.85(1.57)$ & $90.13(8.29)$ & $91.75(12.57)$ & $11.65(33.48)$ & $9.91(8.28)$ \\
$6-8$ & 20 & $99.43(0.51)$ & $99.39(3.93)$ & $0.84(5.72)$ & $0.57(0.51)$ & $80.57(9.30)$ & $86.99(10.65)$ & $13.17(13.99)$ & $19.44(9.28)$ \\
$6-8$ & 10 & $97.32(5.87)$ & $94.13(14.18)$ & $11.39(36.94)$ & $2.68(5.85)$ & $74.37(12.86)$ & $79.34(16.44)$ & $26.61(52.54)$ & $25.66(12.84)$ \\
$9-10$ & 20 & $99.17(0.73)$ & $99.71(10.75)$ & $5.58(20.58)$ & $0.83(0.73)$ & $70.74(11.62)$ & $80.73(12.35)$ & $18.32(15.38)$ & $29.27(11.63)$ \\
\hline
\end{tabular}


shows that the spikes of MU 4 have low energy and their amplitudes are very close to the base line noise, leading to this poorer performance.

The same indexes used for simulated signals were also used to verify the agreement between the responses of the method and the annotations of the human expert. The results (mean (standard deviation)) show the following: (i) correct classification rate: $76.73 \%$ (9.90); (ii) precision: $77.60 \%$ (18.04); (iii) false positive rate: $26.4 \%(28.2)$; and (iv) false negative rate: $23.27 \%(9.90)$.

Figure 6 shows the results of the EMG decomposition provided by the EMGD-MF system for the whole data set (120 seconds). The shimmer plots (with the MUAPs and the average waveform) for each MU are shown on the left. Although the results may also be biased by possible errors made by the expert when marking the MUAPs, the histograms of the IDIs for MUs 1, 2 and 3 follow the expected Gaussian distribution. The mean firing rate for those MUs, calculated as the maximum of their IDI density functions (Kernel Density Estimation), show mean errors between $0.73 \%$ and $1.32 \%$ when these rates are compared to those found by the human expert (vertical red line on the histograms). However, the histogram for MU 4 is not consistent with a Gaussian distribution, most

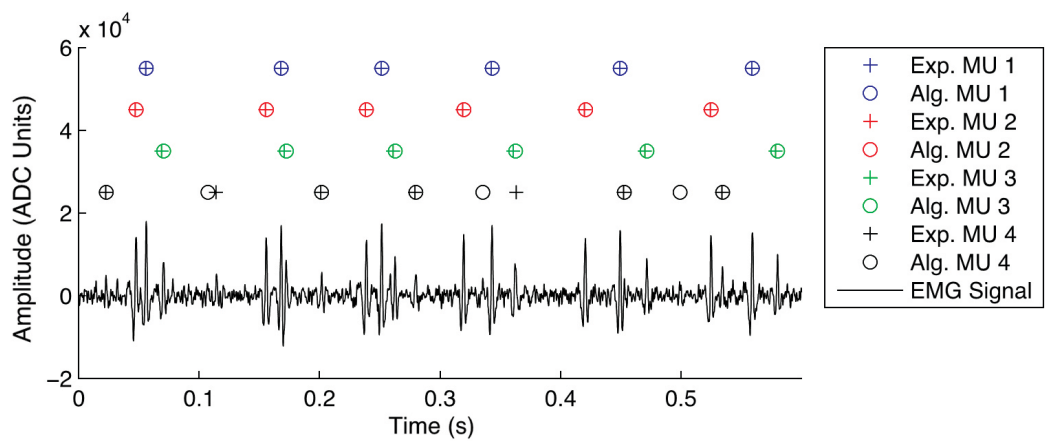

Figure 5. A sample of a real EMG signal and the annotations of MUAPs made by an expert (crosses) versus the results obtained by the EMGD-MF system (circles).
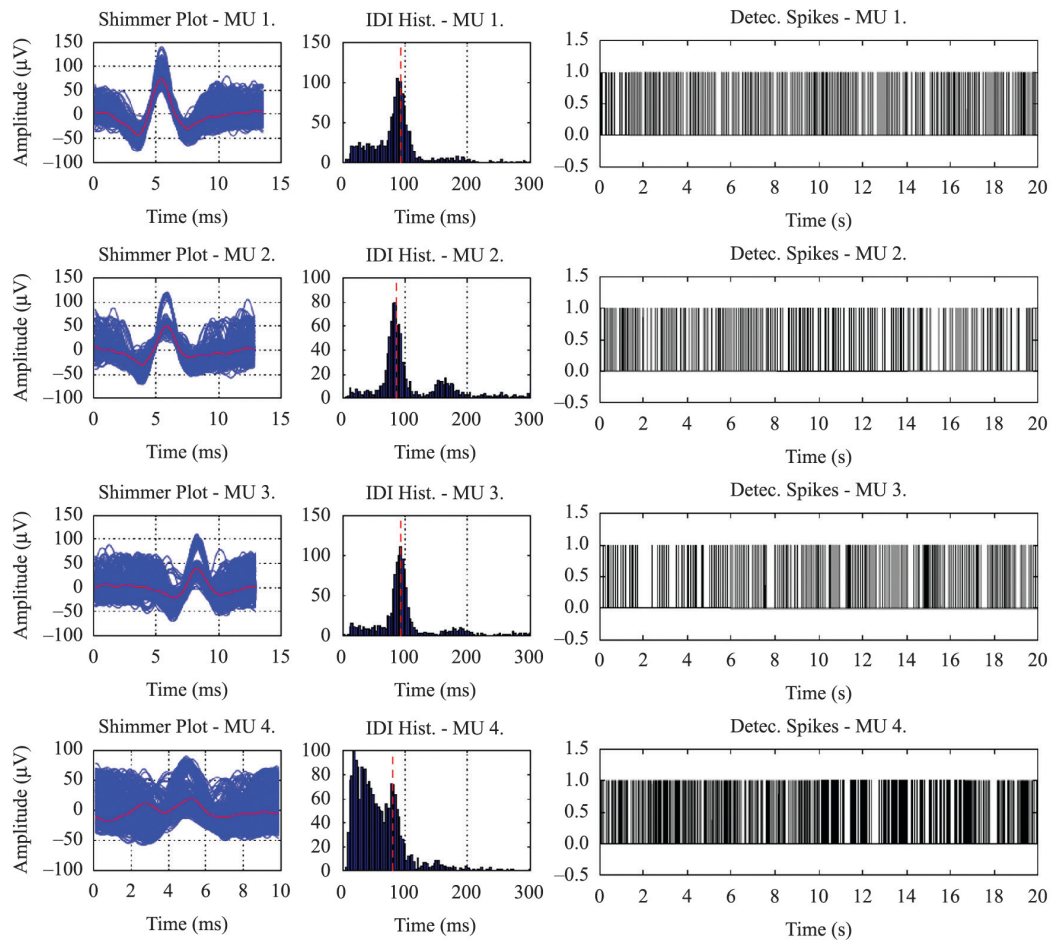

Figure 6. Decomposition of a real EMG signal $(120 \mathrm{sec})$ containing 4 active MUs. The shimmer plot, the histogram of the inter-discharge/ spikes intervals (IDIs) and a sample of the pulse train for each MU are presented in the left, center and right columns, respectively. The vertical dotted red lines on the histograms show the actual average of the IDIs calculated according to the annotations of a human expert. 
likely due to the low amplitude of its MUAPs being close to the noise level of the signal.

The model was implemented in Matlab ${ }^{\circledR}$ (The MathWorks Inc, Natick, Massachusetts, USA) on a desktop computer (Intel ${ }^{\circledR}$ i5 $2.3 \mathrm{GHz}$ Processer, 8 GbGB RAM). On average, $50 \mathrm{~ms}$ windows were processed under $5 \mathrm{~ms}$.

\section{Discussion}

Our results demonstrate that the EMGD-MF method can provide a viable alternative to decompose EMG signals with high accuracy. On average, the method achieved rates of success greater than $97 \%$ when no overlapping was present in simulated signals. Correct classification of up to $92.78 \%$ was achieved for simulated signals with up to 5 overlapping MUs and a $20 \mathrm{~dB}$ SNR. When processing a real surface EMG signal, the method matches $76.73 \%$ of the annotations of a human expert. The histograms of the MUs' firing rates show the expected Gaussian behavior. However, the method has shown some difficulties with low energy MUAPs close to the base noise level. Previous studies have also reported such problems (Parsaei and Stashuk, 2011; Florestal et al., 2009), and a viable solution should rely upon pre-processing techniques to improve the SNR (Andrade et al., 2006; 2007; Sá et al., 2014).

Similar to other strategies for EMG decomposition described in the literature (Florestal et al., 2009; Glaser et al., 2013; Parsaei and Stashuk, 2011; Studer et al., 1984; Winslow et al., 2009), our method also requires previous knowledge of the MUAPs' waveforms to generate a template for each filter. Additionally, at this point, the algorithm is not yet capable of identifying MUAPs different than those previously known. Therefore, this version of the system must be used in cases where the level of muscle contraction remains constant or decreases over time. Note that this is also the case for other works described in the literature (Glaser et al., 2013; Parsaei and Stashuk, 2011). The authors are currently working on strategies to define new filters dynamically as new MUs are recruited during the contraction. The initial approach is based on an EMG segmentation algorithm that runs in parallel with EMGD-MF. The segments and the outputs of the system are continuously monitored to detect segments with features that are too far from the existing templates. Whenever that is the case, a new filter is programmed with a template for that segment and added to the bank. In doing so, the EMGD-MF has the potential to start operation as "empty", being constructed as the signal is processed. A potential impact of this method could be on the maximum speed of execution - as the number of filters increases, the overall computational cost should also increase. Nevertheless, the authors believe that future hardware based on parallel processors (already common in the market) can minimize this effect. This technique is currently under investigation and will be the subject of a future publication.

Because the methods found in the literature for EMG decomposition are considerably different from each other, most of them requiring specific hardware and different techniques for data acquisition (multichannel/array electrode configurations, etc.), direct comparisons are not always possible and could be prone to questioning. However, as we show next, a general comparison based on results obtained when similar data are used (same SNR and same number of active MUs) shows that the performance of the EMGD-MF algorithm matches those of current methods described in the literature.

A number of methods for EMG decomposition have been proposed over the past decade. Although some have shown impressive performance during controlled experiments, there are important limitations that need to be addressed. For instance, Florestal et al. (2009) proposed a method for EMG decomposition that can correctly classify $75 \%$ of the MUAPs present in EMG signals with up to 95\% accuracy. However, when all MUs (12 in this case) are taken into account, the rates of success vary from $53 \%$ to $100 \%$. Furthermore, their best results were found when processing data collected by matrices of electrodes. Recently, Glaser et al. (2013) proposed a system for online MUAP decomposition that uses a preliminary training stage for which a packet of at least 3 seconds of EMG data is required. This method achieves correct classification rates of up to $99 \%$ for signals with SNRs of $20 \mathrm{~dB}$, but for contractions of low intensity and with only 5 active MUs. Furthermore, these results were obtained from signals collected by two grids of monopolar electrodes, each containing $6 \times 5$ electrodes. However, the EMGD-MF algorithm can decompose EMG signals from single channels and achieve high rates of success in similar condition (similar SNR, number of active MUAPs and overlapping). It should also be noted that, similar to our method, the system proposed by Florestal et al. (2009) is not able to detect spikes of MUs that did not appear during the first 3 seconds of training.

Furthermore, our algorithm was designed seeking fast responses for real-time/real-life applications, such as myoelectric prosthesis control. To do so, the authors avoided traditional techniques used for EMG decomposition that rely upon feature extraction and clustering algorithms, which demand extensive 
computation, in favor of a classifier that uses digital filters at its core. In doing so, the architecture of the EMGD-MF system enables the use of a number of computing optimization techniques existing in current digital signal processing systems (DSPs), such as concurrent and parallel processing, which can boost the performance as required. Our experiments were executed in Matlab $₫$ on a standard desktop computer, and on average, a $50 \mathrm{~ms}$ window was processed in under $5 \mathrm{~ms}$.

Current strategies for myoelectric control of prosthetic devices (Masters et al., 2014; Simon et al., 2011) having average delays of up to $100 \mathrm{~ms}$ are considered acceptable for the response time between the user's control (muscle contractions sampled at $25 \mathrm{~ms}-50 \mathrm{~ms}$ windows) and the prosthesis activation. Nevertheless, researchers in the field still face the challenge of choosing the best set of features to be used to achieve dexterous control of advanced prosthetic devices (Masters et al., 2014). The use of traditional features such as waveform envelop, means and zero crossing is limited and cannot extract a great deal of information from within the EMG signal. The authors believe that strategies based on detailed information, such as the motor unit recruitment ratio, may allow for more sophisticated control. However, to be deployed as a viable method, the algorithms for EMG decomposition should be able to run on limited resources (embedded microprocessors/DSPs) and deliver fast response times. Because the EMGD-MF algorithm requires only $5 \mathrm{~ms}$ to process a $50 \mathrm{~ms}$ window (even on a non-optimized system - standard desktop computer running Matlab ${ }^{\circledR}$ ) and encapsulates features that allow for its implementation on standard DSP systems, the authors believe that it has great potential to be applied in future myoelectric control systems based on MUAP activation.

When considering the ability of the EMGD-MF to predict the mean firing rate of different MUAPs, the results show that the system is capable of delivering good estimates, with mean errors below $3.28 \%$. The mean firing rate of MUs is one of the most important results an EMG decomposition system can output because this information allows for clinical use of the technique and is related to the integrity of both the muscle fibers and the neuromotor recruitment pattern (Stashuk, 2001).

As shown, this article contributes to studies on EMG decomposition by describing a novel technique capable of achieving high rates of success under high levels of noise. A limitation of the method is the possibility of false positives due to the presence of MUAPs that are not related to those programmed into the bank of matched filters. A possible solution would require the use of supervised methods in which the response of the classifier is constantly evaluated based on past history and a new filter is dynamically programmed whenever necessary. This work is in progress and will be the subject of future publication.

\section{Acknowledgements}

The authors would like to thank FAPEMIG-MG-Brazil, CNPq-Brazil and CAPES-Brazil for financial support.

\section{References}

Andrade A, Nasuto S, Kyberd P, Sweeney-Reed C, Van Kanijn F. EMG signal filtering based on Empirical Mode Decomposition. Biomedical Signal Processing and Control. 2006; 1(1):44-55. http://dx.doi.org/10.1016/j.bspc.2006.03.003.

Andrade A, Nasuto S, Kyberd P. An automatic system for clustering and visualization of motor unit action potentials based on generative topographic mapping. 3rd IEE International Seminar on Medical Applications of Signal Processing (Ref No 2005-1119). 2005 Nov 3-4. IET; 2005. 125-30.

Andrade AO, Nasuto SJ, Kyberd P. Extraction of motor unit action potentials from electromyographic signals through generative topographic mapping. Journal of the Franklin Institute. 2007; 344(3-4):154-79. http://dx.doi.org/10.1016/j. jfranklin.2006.10.006.

Barboi AC, Barkhaus PE. Electrodiagnostic testing in neuromuscular disorders. Neurologic Clinics. 2004; 22(3):619-41, vi. http://dx.doi.org/10.1016/j.ncl.2004.03.007. PMid: 15207878

Christodoulou C, Pattichis C. A new technique for the classification and decomposition of EMG signals. Proceedings of the IEEE International Conference on Neural Networks. 1995 Nov/Dec; Perth. IEEE; 1995; 5. p. 2303-5.

De Luca CJ, Adam A, Wotiz R, Gilmore LD, Nawab $\mathrm{SH}$. Decomposition of surface EMG signals. Journal of Neurophysiology. 2006; 96(3):1646-57. http://dx.doi. org/10.1152/jn.00009.2006. PMid:16899649

De Luca CJ, Hostage EC. Relationship between firing rate and recruitment threshold of motoneurons in voluntary isometric contractions. Journal of Neurophysiology. 2010; 104(2):1034-46. http://dx.doi.org/10.1152/jn.01018.2009. PMid:20554838

Fang J, Agarwal GC, Shahani BT. Decomposition of multiunit electromyographic signals. IEEE Transactions on Bio-Medical Engineering. 1999; 46(6):685-97. http:// dx.doi.org/10.1109/10.764945. PMid:10356875

Florestal JR, Mathieu PA, Malanda A. Automated decomposition of intramuscular electromyographic signals. IEEE Transactions on Bio-Medical Engineering. 2006; 53(5):832-9. http://dx.doi.org/10.1109/TBME.2005.863893. PMid:16686405

Florestal JRF, Mathieu PAP, McGill KCK. Automatic decomposition of multichannel intramuscular EMG signals. Journal of electromyography and kinesiology : official 
journal of the International Society of Electrophysiological Kinesiology. 2009; 19(1):1-9. http://dx.doi.org/10.1016/j. jelekin.2007.04.001. PMid:17513128

Glaser V, Holobar A, Zazula D. Real-time motor unit identification from high-density surface EMG. IEEE transactions on neural systems and rehabilitation engineering : a publication of the IEEE Engineering in Medicine and Biology Society. 2013; 21(6):949-58. http://dx.doi.org/10.1109/ TNSRE.2013.2247631. PMid:23475379

Han JJ, Carter GT, Weiss MD, Shekar C, Kornegay JN. Using electromyography to assess function in humans and animal models of muscular dystrophy. Physical Medicine and Rehabilitation Clinics of North America. 2005; 16(4):981-97, x. http://dx.doi.org/10.1016/j.pmr.2005.08.001. PMid:16214055

Hogrel JY. EMGLAB: A forum for sharing software, data, and information related to EMG decomposition. Online dataset R011. 2014. [cited: 30 july 2014]. Available at: http://www.emglab.net.

Holobar A, Farina D, Gazzoni M, Merletti R, Zazula D. Estimating motor unit discharge patterns from high-density surface electromyogram. Clinical neurophysiology : official journal of the International Federation of Clinical Neurophysiology. 2009; 120(3):551-62. http://dx.doi. org/10.1016/j.clinph.2008.10.160. PMid:19208498

Kleine BU, Boekestein WA, Arts IM, Zwarts MJ, Schelhaas HJ, Stegeman DF. Fasciculations and their F-response revisited: high-density surface EMG in ALS and benign fasciculations. Clinical neurophysiology : official journal of the International Federation of Clinical Neurophysiology. 2012; 123(2):399-405. http://dx.doi.org/10.1016/j. clinph.2011.06.032. PMid:21820354

Lathi BP, Ding Z. Modern digital and analog communication systems. 4th ed. New York: Oxford University Press USA; 2009.

LeFever RS, De Luca CJ. A procedure for decomposing the myoelectric signal into its constituent action potentials - Part I: technique, theory, and implementation. IEEE Transactions on Bio-Medical Engineering. 1982; 29(3):149-57. http:// dx.doi.org/10.1109/TBME.1982.324881. PMid:7084948

Mambrito B, De Luca CJ. A technique for the detection, decomposition and analysis of the EMG signal. Electroencephalography and Clinical Neurophysiology. 1984; 58(2):175-88. http://dx.doi.org/10.1016/00134694(84)90031-2. PMid:6204844

Marateb HR, Muceli S, McGill KC, Merletti R, Farina D. Robust decomposition of single-channel intramuscular EMG signals at low force levels. Journal of Neural Engineering. 2011; 8(6):066015. http://dx.doi.org/10.1088/17412560/8/6/066015. PMid:22063475

Masters MR, Smith RJ, Soares AB, Thakor NV. Towards better understanding and reducing the effect of limb position on myoelectric upper-limb prostheses. EMBC 2014: Proceedings of the 36th Annual International Conference of the IEEE Engineering in Medicine and Biology Society; 2014 Aug 26-30; Chicago. 2014. p. 2577-80.
McGill KC, Cummins KL, Dorfman LJ. Automatic decomposition of the clinical electromyogram. IEEE Transactions on Bio-Medical Engineering. 1985; 32(7):470-7. http://dx.doi.org/10.1109/TBME.1985.325562. PMid:3839488

McGill KC, Marateb HR. Rigorous a posteriori assessment of accuracy in EMG decomposition. IEEE transactions on neural systems and rehabilitation engineering: a publication of the IEEE Engineering in Medicine and Biology Society. 2011; 19(1):54-63. http://dx.doi.org/10.1109/TNSRE.2010.2056390. PMid:20639182

Nawab SH, Wotiz RP, De Luca CJ. Decomposition of indwelling EMG signals. Journal of Applied Physiology. 2008; 105(2):700-10. http://dx.doi.org/10.1152/ japplphysiol.00170.2007. PMid:18483170

Parsaei H, Stashuk DW, Rasheed S, Farkas C, HamiltonWright A. Intramuscular EMG signal decomposition. Critical Reviews in Biomedical Engineering. 2010; 38(5):435-65. http://dx.doi.org/10.1615/CritRevBiomedEng.v38.i5.20. PMid:21175408

Parsaei H, Stashuk DW. Adaptive motor unit potential train validation using MUP shape information. Medical Engineering \& Physics 2011; 33(5):581-9. http://dx.doi. org/10.1016/j.medengphy.2010.12.012. PMid:21269867

Parsaei H, Stashuk DW. EMG signal decomposition using motor unit potential train validity. IEEE transactions on neural systems and rehabilitation engineering: a publication of the IEEE Engineering in Medicine and Biology Society. 2013; 21(2):265-74. http://dx.doi.org/10.1109/TNSRE.2012.2218287. PMid:23033332

Pino LJ, Stashuk DW, Boe SG, Doherty TJ. Motor unit potential characterization using "pattern discovery". Medical Engineering \& Physics. 2008; 30(5):563-73. http://dx.doi. org/10.1016/j.medengphy.2007.06.005. PMid:17697793

Rasheed S, Stashuk D, Kamel M. A software package for interactive motor unit potential classification using fuzzy k-NN classifier. Computer Methods and Programs in Biomedicine. 2008; 89(1):56-71. http://dx.doi.org/10.1016/j. cmpb.2007.10.006. PMid:18054118

Ren X, Hu X, Wang Z, Yan Z. MUAP extraction and classification based on wavelet transform and ICA for EMG decomposition. Medical \& Biological Engineering \& Computing. 2006; 44(5):371-82. http://dx.doi.org/10.1007/ s11517-006-0051-3. PMid:16937179

Sá AAR, Soares AB, Andrade AO, Nasuto S. Decomposition of surface electromyographic signal using Hidden Markov Model. Journal of Health Science. 2014; 2:28-40.

Simon AM, Hargrove LJ, Lock BA, Kuiken TA. Target Achievement Control Test: evaluating real-time myoelectric pattern-recognition control of multifunctional upperlimb prostheses. Journal of Rehabilitation Research and Development. 2011; 48(6):619-27. http://dx.doi.org/10.1682/ JRRD.2010.08.0149. PMid:21938650

Stashuk D. EMG signal decomposition: how can it be accomplished and used? Journal of electromyography and kinesiology : official journal of the International Society of Electrophysiological Kinesiology. 2001; 11(3):151- 
73. http://dx.doi.org/10.1016/S1050-6411(00)00050-X. PMid:11335147

Studer RM, de Figueiredo RJP, Moschytz GS. An algorithm for sequential signal estimation and system identification for EMG signals. IEEE Transactions on Bio-Medical Engineering. 1984; 31(3):285-95. http://dx.doi.org/10.1109/ TBME.1984.325267. PMid:6546925

Turin G. An introduction to matched filters. I.R.E. Transactions on Information Theory. 1960; 6(3):311-29. http://dx.doi. org/10.1109/TIT.1960.1057571.

Winslow J, Dididze M, Thomas CK. Automatic classification of motor unit potentials in surface EMG recorded from thenar muscles paralyzed by spinal cord injury. Journal of
Neuroscience Methods. 2009; 185(1):165-77. http://dx.doi. org/10.1016/j.jneumeth.2009.09.012. PMid:19761794

Xu Z, Xiao S, Chi Z. ART2 neural network for surface EMG decomposition. Neural Computing \& Applications. 2001; 10(1):29-38. http://dx.doi.org/10.1007/s005210170015.

Zhou P, Barkhaus PE, Zhang X, Rymer WZ. Characterizing the complexity of spontaneous motor unit patterns of amyotrophic lateral sclerosis using approximate entropy. Journal of Neural Engineering. 2011; 8(6):066010. http:// dx.doi.org/10.1088/1741-2560/8/6/066010. PMid:22049095

Zijdewind I, Thomas CK. Firing patterns of spontaneously active motor units in spinal cord-injured subjects. The Journal of Physiology. 2012; 590(Pt 7):1683-97. http:// dx.doi.org/10.1113/jphysiol.2011.220103. PMid:22310313

\section{Authors}

Ailton Luiz Dias Siqueira Júnior ${ }^{1,2 *}$, Alcimar Barbosa Soares ${ }^{1}$

${ }^{1}$ Faculty of Electrical Engineering, Federal University of Uberlândia - UFU, Campus Santa Mônica, Bloco 1E, CEP 38400-902, Uberlândia, MG, Brazil.

${ }^{2}$ Federal Institute of the Triângulo Mineiro, Ituiutaba, MG, Brazil. 